\title{
Internet of Things for Dementia Care
}

\author{
Shirin Enshaeifar ${ }^{1}$, Payam Barnaghi ${ }^{1}$, Severin Skillman ${ }^{1}$, Andreas Markides ${ }^{1}$, Tarek Elsaleh ${ }^{1}$, Thomas Acton ${ }^{1}$, \\ Ramin Nilforooshan ${ }^{2}$, and Helen Rostill ${ }^{2}$ \\ ${ }^{1}$ Department of Electrical and Electronic Engineering, University of Surrey, UK. \\ ${ }^{2}$ Surrey and Borders Partnership NHS Foundation Trust, Leatherhead, Surrey, UK.
}

\begin{abstract}
In this paper we discuss a technical design and an ongoing trial that is being conducted in the UK, called Technology Integrated Health Management (TIHM). TIHM uses Internet of Things (IoT) enabled solutions provided by various companies in a collaborative project. The IoT devices and solutions are integrated in a common platform that supports interoperable and open standards. A set of machine learning and data analytics algorithms generate notifications regarding the well-being of the patients. The information is monitored around the clock by a group of healthcare practitioners who take appropriate decisions according to the collected data and generated notifications. In this paper we discuss the design principles and the lessons that we have learned by co-designing this system with patients, their carers, clinicians, and also our industry partners. We discuss the technical design of TIHM and explain why user-centred and human-experience should be an integral part of the technological design.
\end{abstract}

Index Terms-Internet of Things (IoT), Integrated Systems, Healthcare, Dementia.

\section{INTRODUCTION}

Dementia affects more than 46 million people around the world. In the UK there are currently around 850,000 dementia patients and this number is estimated to grow to 1 million by 2025 [1].

Recent advancements in the design of Internet of Things (IoT) have led to the development of smart systems [2] and connected worlds of physical objects, people, and devices which are significantly affecting the human experience and the way we interact with our surrounding environment. For example, several works have focused on the establishment of smartcities [2]-[5], smart-homes [6]-[9], and smart-healthcare [10][13]. These are amongst the most important and challenging scenarios where IoT platforms are changing the daily human experience.

In particular, smart systems can support and improve healthcare-related processes to deliver quality care to patients, while reducing healthcare costs and tackling the nursing staff shortage problem for larger ageing populations. According to [12], current procedures for patient monitoring, care, management, and supervision are often manually executed by healthcare practitioners. Therefore, this study exploits an advanced IoT platform to propose a technology integrated health management (TIHM) ${ }^{1}$ system which is developed based on different, yet complementary, technologies. This integration

\footnotetext{
1 'TIHM for Dementia' is a study led by Surrey and Borders Partnership and funded by the Department of Health.
}

facilitates the development of an automatic monitoring system which assists the healthcare practitioners to support dementia patients and their carers. As well as delivering quality of care to patients, the intention is to reduce carer stress and burden in order to prolong the time patients live in their own homes, while improving their quality of life. TIHM allows to obtain real-time insights into the health status of people with dementia. Note that, traditionally, medical history relies on anecdotal accounts and memory between clinic visits. However, our IoT platform provides an immediacy for the healthcare practitioner which will improve clinical decision making and ensure the right level of support can be deployed at the earliest point of need to prevent escalating crisis.

This project has brought together a team of clinical and healthcare experts, small and medium-sized enterprises (SMEs) with IoT products/services, and academic groups with healthcare, economy, security, and technical expertise. Note that this paper is involved with the technical aspects and focuses on development of the physical infrastructure and advanced analytical techniques for the IoT testbed. TIHM uses a combination of passive environmental sensors, medical devices, wearable technologies, and interactive applications to collect real-time data containing information on environmental conditions, patients physiological parameters, and their daily lifestyles.

In general, efficient access and use of IoT data are highly dependent on quality, latency, availability, reliability, and continuity of the data and services [14]; where some of these factors correspond to specific challenges in terms of big data analysis, such as volume, variety, velocity, or veracity. Similarly, in the TIHM system, we have a dynamic and complicated ecosystem in terms of the number of resources, heterogeneity and complexity, and the amount of data. In other words, we are facing the following challenges:

- Volume; This project is recruiting up to 700 patients, with mild to moderate dementia, where half of them will be considered as a control group and the other half will receive all the technologies. This includes approximately 25 sensors/apps per home, where some of them provide stream measurements; leading to the generation of large volume of data in the TIHM project.

- Velocity; One of the aims of TIHM is to enable continuous monitoring and provide real-time response mechanisms in healthcare system. Thus, collecting real-time data and producing online and actionable information for 
the clinical monitoring team ${ }^{2}$ is an essential requirement for this project; i.e. dealing with the velocity challenge.

- Variety; This project involves different types of measurements originating from various sources, such as passive sensors, medical devices, wearable technologies, and interactive application. Furthermore, it should be considered that devices used in TIHM are provided by several different industrial partners using their in-house technologies and standards which would lead to different publishing methods and/or formats.

Given the above challenges, this work briefly describes the technical efforts and activities conducted in the early stages of the TIHM project to resolve these issues. Note that, to this point, our IoT testbed has been already deployed and evaluated in real-world scenarios, including two "living labs" and seven "trusted patient homes". The next phase of this project will focus on the main deployment in a larger scale (up to 350 homes) and development of innovative data analysis tools and advanced machine learning algorithms.

\section{TIHM INFRASTRUCTURE}

This section describes the design and implementation of our IoT architecture which is capable of combining different, yet complementary, technologies to develop novel functionalities. Our system collects real-time data from multiple publishers and delivers the data to the TIHM backend system. At this stage, an advanced analytics tool is used to process the integrated data and generate insights and notifications based on the status of patients. Note that clinical knowledge and experience has been used to set the parameters for alerting and notifications.

The high-level overview of the interactions in TIHM architecture is presented in Fig. 1. As shown, it is composed of four main parts: 1) sensors installed at homes, 2) SMEs' back-end servers, 3) TIHM backend system including the storage and analysis servers, and 4) the user interface for data visualisation and management.

At a finer level of detail, several passive sensors are embedded in patients' homes to collect data from the environment and surroundings, such as humidity and temperature conditions, appliance usage, etc. In addition, medical devices and wearable technologies are used to measure important physiological parameters, such as blood pressure, pulse, etc. All the sensor and medical devices record and send the data to their corresponding gateways over Wi-Fi, Bluetooth or, in exceptional cases, via auxiliary interfaces of such a device. Gateways relay these data to the companies backend systems over GPRS, SigFox or home broadband. At this stage, all participating companies comply with a common JSON data model for the TIHM project which is defined in Section III. This is followed by the communication with the TIHM backend system through a publish/subscribe (pub/sub) or message queue (MQ) model, specifically the advanced message queuing protocol (AMQP) is employed. These data are then validated

\footnotetext{
${ }^{2}$ The clinical monitoring team is responsible for continuous monitoring of the available data and generated alerts.
}

and persisted to a NoSQL Mongo database for further analysis. To make the analysed data more readily accessible and consumable by remote users (mainly the clinical monitoring team), a web-based graphical interface has been implemented with defined privilege levels to access both real-time and historical data [12] - comprehensive details are included in Section V. Observe that the MQ is the core exchange for TIHM messages and is able to receive, process, and reply to requests coming from the companies back-ends, TIHM backend system, and the user interface.

Since the data produced in TIHM are created from real patients' homes, privacy and security, ownership, duration of storage, and types of use are key concerns in this project. To address these, we have followed the following approaches:

- Observation resources are anonymised and sensitive data, such as GPS locations, are always encrypted before persisting to storage.

- The servers are physically secured and they comply with NHS standards on secure data storage, e.g. communication with the monitoring team is via the NHS's private secure network.

- All company vendors establish a connection with the server over a mutually authenticated encrypted channel with certificate exchange.

- Once authenticated, each company is authorised to publish and subscribe to their own exchange/queues. Note that interaction with the TIHM back-end is restricted through firewall rules.

- The user interface enforces authentication and role-based authorisation for administration, deployment, clinical, technical, and other roles corresponding to their respective work streams.

- There is also an implemented audit trail for the user interface and message validation back-end.

Following the above steps, we collect the real-time data from all publishers and store them in a secure database with nightly backups. To allow the integration of these data, a common data model has been specifically defined for this project, see next section.

\section{DATA INTEROPERABILITY AND INTEGRATION}

The collected data in the TIHM project originate from different technologies (such as environmental and medical sensors, wearable technologies and interactive applications) and published by multiple providers, meaning that the original data may have different formats (e.g. CSV, XML, or JSON). Having such a wide range of data provided by the heterogeneous, ubiquitous, and dynamic nature of the devices/sensors presents a challenge in accessing, processing, integrating, and interpreting the data [15]. Thus, it is important to define a common data model to provide the interoperability and allow for the integration of multi-modal data.

To this end, we have adapted Health Level-7 (HL7) which refers to a set of international standards for the transfer of clinical and administrative data between software applications used by various healthcare providers. HL7 specifies a number of flexible standards, guidelines, and methodologies by which 


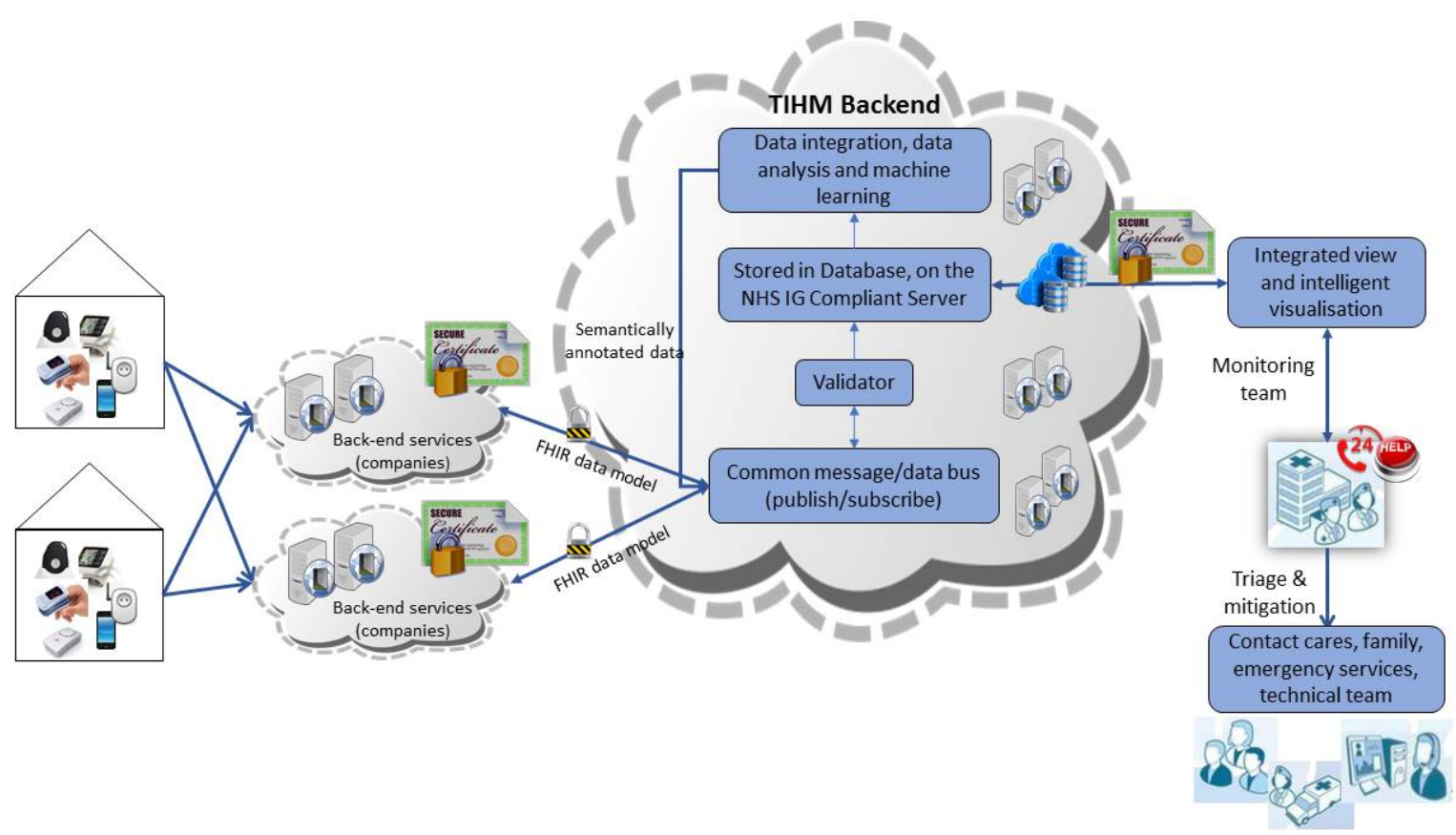

Fig. 1: The high-level overview of the interactions in TIHM architecture.

various healthcare systems can communicate with each other. In particular, the Fast Healthcare Interoperability Resources (FHIR) is a new standard derived from HL7 designed to be easier to implement, more open, and more extensible than other versions of HL7. FHIR makes use of an HL7-defined set of "resources" to support information sharing by a variety of means, including documents, messages, etc. This makes it suitable for use in a wide variety of contexts, such as mobile phone apps, cloud communications, and server communication in large institutional healthcare providers [16].

In the same spirit, a subset of FHIR resources have been selected for the TIHM project to provide the interoperability. Since the collected data in TIHM cover a wide range of measurements, and not just clinical, it was necessary to adapt the FHIR model so that it complies with TIHM requirements - this led to the development of FHIR4TIHM ${ }^{3}$ data model. The main FHIR resources used in this project are 'Patient', 'Device', 'Observation', 'QuestionnaireResponse', 'Flag', and 'DetectedIssue'. Additional resources were also created to facilitate the needs of this project. All resources are reusable structures where each resource has logical table, UML definition, block diagram, and XML or JSON template, see Fig 2.

With data consistency in mind across data publishers, validation of the incoming data must be strict. To this end, a JSON validator (schema) has been prepared according to the agreed data model and set up on the central TIHM backend to validate the incoming data. Accordingly, if the data received on the TIHM server is invalid, the corresponding publisher would be notified about the failed validation and the reasons of invalidity. Otherwise, the valid data would be stored in a database for ensuing analysis - which is addressed in the

\footnotetext{
${ }^{3}$ Full description of FHIR4TIHM data model is available at http://iot.ee.surrey.ac.uk/tihm/models/fhir4tihm.
}

following section.

\section{Data Governance And AnAlysis}

Generally, real-world data should undergo pre-processing, analysis, and interpretation to provide meaningful information and actionable knowledge. Similarly, given the real-time and dynamic healthcare data in TIHM, creating human- and/or machine-understandable information from raw observations and providing real-time response mechanisms are the main data analytics challenges in this project. To initiate the data analysis, the integrated data were first validated to comply with the FHIR4TIHM data model and then stored in the Mongo database.

In the next step, the integrated datasets were processed using several data analysis and machine learning algorithms to generate appropriate notifications based on patients' needs aligned to the parameters set by the clinical team. TIHM is a clinical trial project which will involve up to 700 dementia patients. The data analytics algorithms aim to provide early indication of patients' needs allowing the clinical team to provide a timely response and prevent escalating ill health.

To this point, in order to analyse the data, we have queried the database and applied several algorithms, such as cleaning, aggregation, and filtering to pre-process the raw data. In addition, further methods including abstraction, pattern detection, machine learning, and adaptive thresholding have been employed to extract meaningful information and to provide interpretation for (near) real-time analytical processing. This way, the raw data from various sources were integrated, processed, and interpreted to supply actionable knowledge and improve the decision making. This, accordingly, enhanced the human experience in healthcare and targeted resources towards need at an earlier stage. 


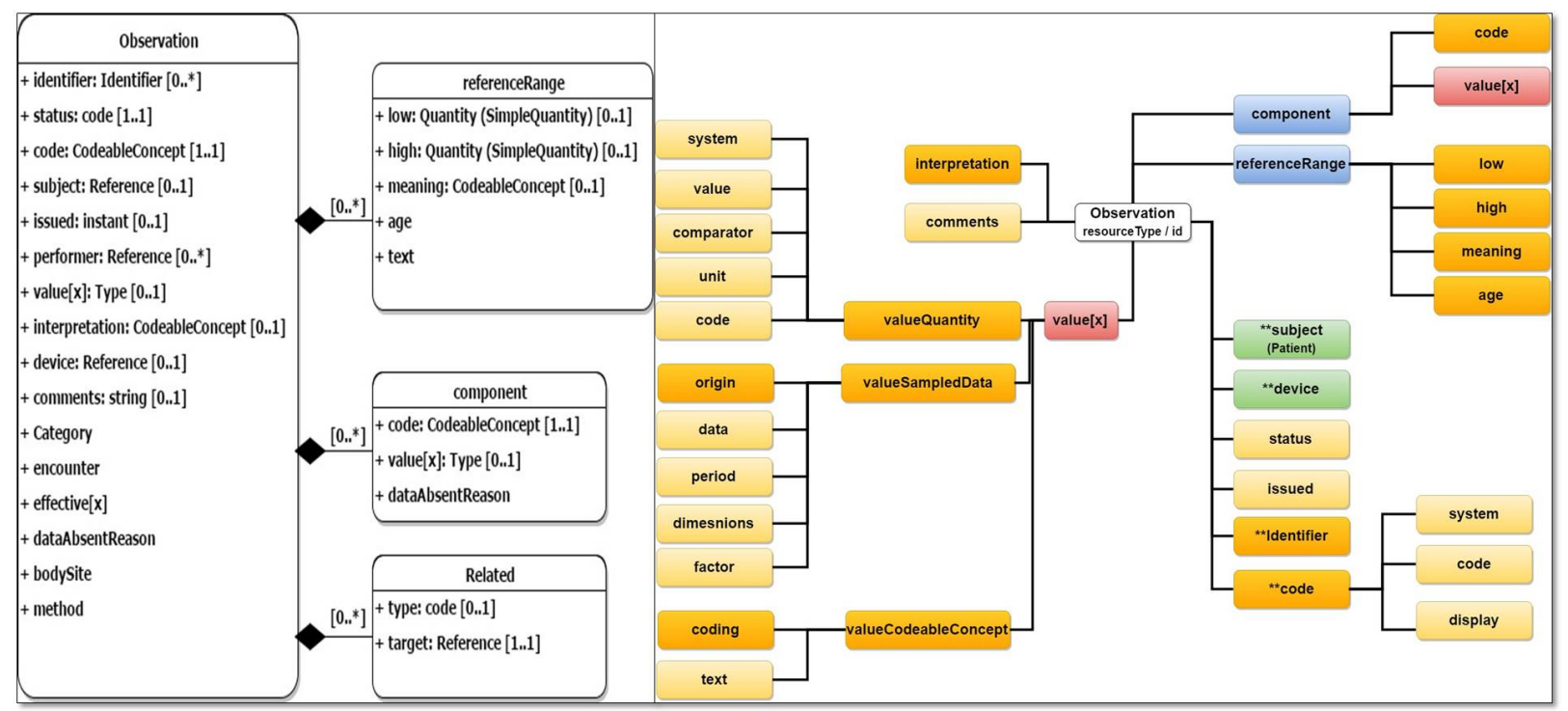

Fig. 2: An example of the observation resource defined in the FHIR4TIHM data model. The UML definition and block diagram of the observation resource are shown in (left) and (right), respectively.

The output of our current data analytics tool is composed of (i) "Reminder texts" for patients, and (ii) "Flag resources" for companies and the monitoring team:

Reminder; The reminders will be generated if a pre-planned measurement is missing and/or incomplete ${ }^{4}$. These messages are generated by the machine learning algorithms and published to the MQ. Thus, corresponding companies could notify patients and their carers about re-assessments using the interactive applications.

Flag; To this point, our generated flags cover three types of notifications, including 'Clinical', 'Environmental', and 'Technical'. Note that each flag resource contains three main components to provide comprehensive information about: (i) the corresponding source of measurement, i.e. the user (or device where applicable), (ii) the pre-set standard thresholds for that measurement and an informative message for the monitoring team, and also (ii) the severity of flag which is defined in terms of percentage. Once a flag has been generated, the monitoring team take appropriate actions according to its type and severity.

The following is the list of goals which have been achieved through analysing the acquired data in the early stages of TIHM project.

- Developing rule-based reasoning algorithms for vital measurements ${ }^{5}$ and providing adaptive learning to make them more personalised. This was used to generate clinical flags for the monitoring team.

- Monitoring the battery level and connectivity status of the implemented sensors for generation of technical flags.

- Detecting the daily routine using the integrated data recorded from passive environmental sensors and wear-

\footnotetext{
${ }^{4}$ In case of an incomplete measurement, the analytics tool generates a reminder assigned to the corresponding patient identifier. This reminder gets published to the MQ and, accordingly, shown on the individual's interactive application.

${ }^{5}$ The rule-based reasoning was performed according to the NHS guidelines for dementia and it was approved by the clinical team.
}

able technologies. This is particularly important to generate environmental and/or complicated clinical flags, such as excessive movements.

- Developing predictive models using a combination of sources, e.g. environmental and medical sensors. For instance, we could inform the clinical team about the potential Urinary Tract Infection (UTI) by monitoring the body temperature and the number of times patient use the bathroom. However, in an isolated environment, UTI can be only diagnosed once the patient refers for clinical assessments.

- Contacting the patient and/or carer on emergency states, such as falls and wandering reported by fall detector and GPS trackers, respectively.

- Providing easy-to-follow audio instruction manuals and reminders for missing and/or incomplete measurements.

- Monitoring the daily mood state (specifically the anxiety level) using the subjective questionnaires designed for TIHM project.

To distinctly display the above information and allow for real-time response mechanisms, we have designed a specific user interface for TIHM project, which is discussed below.

\section{USER INTERFACE}

After analysing the data, the generated notifications, along with the cohort data, are presented on a user interface, the socalled integrated view (iView), to facilitate real-time response mechanism and easy monitoring. This is achieved by querying the database every 20 seconds to gather real-time flags, observations and other resources. Note that the iView is designed and modified according to the clinical team's requirements and a role-based authorisation system is used to provide secure access to the iView $^{6}$. The main pages of our user interface are described below.

\footnotetext{
${ }^{6}$ There are separate roles for users from the monitoring, deployment and the technical teams. However, patients may not access the iView.
} 
1) Overview; All the registered patients are presented using a "symbolic traffic light" notification system. Hence, patients' list gets sorted according to the number of active notifications, their types and severities, as shown in Fig. 3 (a).

2) Individual; Once a specific patient is selected by the monitoring team, they will be navigated to the patient's page which contains his/her details, contact information, list of the active notifications, and latest observations for each category of sensors, see Fig. 3 (b).

3) Flags and Follow up; In order to respond to a flag, a member of the monitoring team clicks on it and fills in the mitigation form to state what action has been taken based on the emergency level. This process will clear the selected flag and create an active follow up ${ }^{7}$ so that the monitoring team track the situation.

4) Management; This page provides brief information about patients, devices, users of the iView, flags, and follow ups. This page also enables the user to view further details and edit a resource, and in some cases deactivate it (depending on the user access right and the type of resource).

\section{Conclusions}

It is estimated that there will be one million people with dementia in the UK by 2025 [1]. Beyond those directly affected, dementia has a much wider impact on carers, families, and health/care services, with social isolation being a key confounding factor. TIHM aims to develop innovative living environments which empower people with dementia and their carers to enjoy better health and quality of life, with reduced dependence on institutional care. One of the key aspects of this project is that user-led design principles have been at the heart of developing the system, allowing us to draw expertise and experience from people with dementia, carers, clinicians, academics, and industry partners. Furthermore, we focus on integrating various data from different providers. In this regard, we have developed a flexible system to integrate the existing heterogeneous data and facilitate the easy integration of other publishers for further expansion because of our semantic interoperability. In addition, we have applied advanced algorithms to analyse the acquired multi-modal data, extract meaningful information, and generate online notifications for caregivers. One of the key features of the machine learning and data analytics algorithms in TIHM is that they combine environmental and physiological data to learn and discover changes in patients' health and well-being. To this point, a number of algorithms have been developed to (i) learn the patients' daily patterns and find possible pattern deviations, (ii) detect if patients are agitated and/or irritated, and (iii) detect the possibility of Urinary Tract Infections (UTI). This provides early identification of need which has the potential to enable smart deployment of healthcare resources, better quality of care, and savings to the health and care economies. TIHM is an ongoing clinical trial which went live in March 2017. The

\footnotetext{
${ }^{7}$ To represent the follow up using the FHIR4TIHM data model, 'DetectedIssue' has been used as the resource.
}

designed interface is being used in Chertsey Hospital (Surrey, England) where the information is monitored around the clock by a team of healthcare practitioners. The empirical evaluation and the healthcare outcomes of TIHM will be published by the end of this trial.

\section{ACKNOWLEDGMENT}

This project is supported by a grant from the Department of Health and NHS England. We would like to thank our project partners and sponsors for their contributions to the project. A full list of the contributors and partners is available at http://www.sabp.nhs.uk/tihm/partnership.

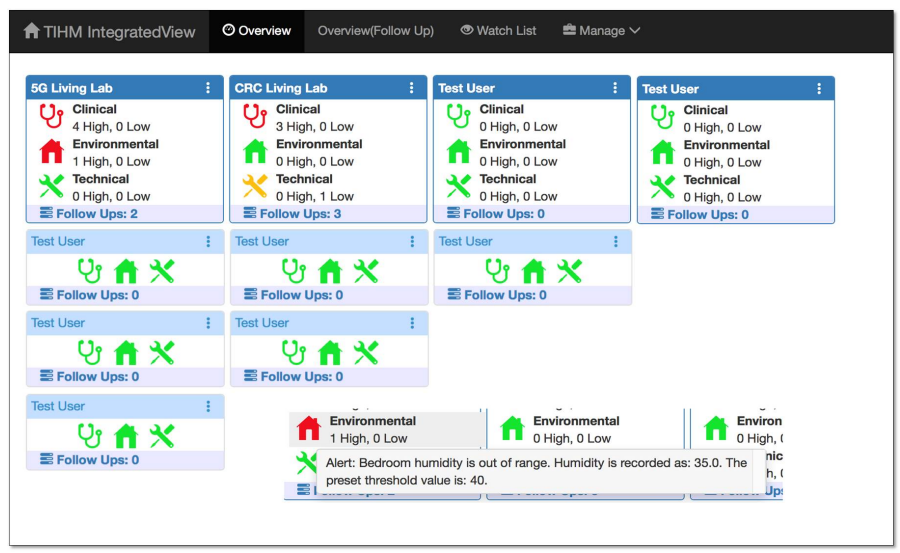

(a) Overview Page

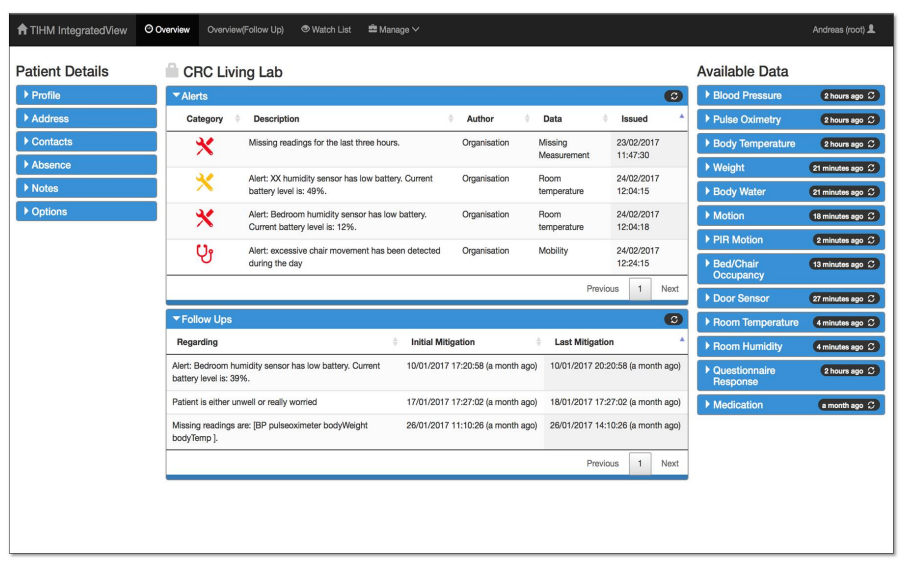

(b) Individual Patient's Page

Fig. 3: Representation of TIHM integrated view.

\section{REFERENCES}

[1] "Alzheimer's Society: dementia support and research charity," https://www.alzheimers.org.uk/, Accessed: 2017-03-30.

[2] J. Gubbi, R. Buyya, S. Marusic, and M. Palaniswami, "Internet of Things (IoT): A vision, architectural elements, and future directions," Future Generation Computer Systems, vol. 29, no. 7, pp. 1645-1660, 2013.

[3] L. Sanchez, L. Muñoz, J. A. Galache, P. Sotres, J. R. Santana, V. Gutierrez, R. Ramdhany, A. Gluhak, S. Krco, E. Theodoridis et al., "Smart Santander: IoT experimentation over a smart city testbed," Computer Networks, vol. 61, pp. 217-238, 2014.

[4] J. Jin, J. Gubbi, S. Marusic, and M. Palaniswami, "An information framework for creating a smart city through Internet of Things," IEEE Internet of Things, vol. 1, no. 2, pp. 112-121, 2014. 
[5] R. Petrolo, V. Loscrì, and N. Mitton, "Towards a smart city based on cloud of things, a survey on the smart city vision and paradigms," Transactions on Emerging Telecommunications Technologies, pp. 1-11, 2015.

[6] G. Demiris, B. K. Hensel et al., "Technologies for an aging society: a systematic review of 'smart home' applications," Yearbook of Medical Informatics, vol. 3, pp. 33-40, 2008.

[7] Q. Ni, A. B. García Hernando, and I. P. de la Cruz, "The elderlys independent living in smart homes: A characterization of activities and sensing infrastructure survey to facilitate services development," Sensors, vol. 15, no. 5, pp. 11312-11362, 2015.

[8] M. R. Alam, M. B. I. Reaz, and M. A. M. Ali, "A review of smart homes - past, present, and future," IEEE Transactions on Systems, Man, and Cybernetics, Part C (Applications and Reviews), vol. 42, no. 6, pp. 1190-1203, 2012.

[9] D. Cook, M. Schmitter-Edgecombe, A. Crandall, C. Sanders, and B. Thomas, "Collecting and disseminating smart home sensor data in the CASAS project," Proceedings of the CHI Workshop on Developing Shared Home Behavior Datasets to Advance HCI and Ubiquitous Computing Research, pp. 1-7, 2009.

[10] C. Doukas and I. Maglogiannis, "Bringing IoT and cloud computing towards pervasive healthcare," Proceedings of IEEE International Conference on Innovative Mobile and Internet Services in Ubiquitous Computing (IMIS), pp. 922-926, 2012.

[11] G. Yang, L. Xie, M. Mäntysalo, X. Zhou, Z. Pang, L. Da Xu, S. KaoWalter, Q. Chen, and L.-R. Zheng, "A health-IoT platform based on the integration of intelligent packaging, unobtrusive bio-sensor, and intelligent medicine box," IEEE Transactions on Industrial Informatics, vol. 10, no. 4, pp. 2180-2191, 2014.

[12] L. Catarinucci, D. De Donno, L. Mainetti, L. Palano, L. Patrono, M. L. Stefanizzi, and L. Tarricone, "An IoT-aware architecture for smart healthcare systems," IEEE Internet of Things, vol. 2, no. 6, pp. 515-526, 2015.

[13] S. Amendola, R. Lodato, S. Manzari, C. Occhiuzzi, and G. Marrocco, "RFID technology for IoT-based personal healthcare in smart spaces," IEEE Internet of Things, vol. 1, no. 2, pp. 144-152, 2014.

[14] P. Barnaghi and A. Sheth, "On searching the Internet of Things: Requirements and challenges," IEEE Intelligent Systems, vol. 31, no. 6 , pp. 71-75, 2016.

[15] P. Barnaghi, A. Sheth, and C. Henson, "From data to actionable knowledge: Big data challenges in the web of things [guest editors' introduction]," IEEE Intelligent Systems, vol. 28, no. 6, pp. 6-11, 2013.

[16] "HL7 FHIR: HL7 Fast Healthcare Interoperability Resources," https://www.hl7.org/fhir/, Accessed: 2017-03-30. 